\title{
Comprehensive identification of essential pathways and transcription factors related to epilepsy by gene set enrichment analysis on microarray datasets
}

\author{
$\mathrm{KAN} \mathrm{HE}^{1 *}$, WEIZHONG XIAO ${ }^{2 *}$ and WENWEN LV ${ }^{1}$ \\ ${ }^{1}$ Center for Stem Cell and Translational Medicine, School of Life Sciences, Anhui University, Hefei, Anhui 230601; \\ ${ }^{2}$ Department of Neurology, Shanghai Pudong Hospital, Fudan University Pudong Medical Center, \\ Pudong, Shanghai 201399, P.R. China
}

Received May 12, 2014; Accepted June 30, 2014

DOI: $10.3892 / \mathrm{ijmm} .2014 .1843$

\begin{abstract}
Epilepsy is a common chronic neurological disorder characterized by seizures or convulsions, and is known to affect patients with primary brain tumors. The etiology of epilepsy is superficially thought to be multifactorial; however, the genetic factors which may be involved in the pathogenesis of seizures have not yet been elucidated, particularly at the pathway level. In the present study, in order to systematically investigate the gene regulatory networks involved in epilepsy, we employed a microarray dataset from the public database library of Gene Expression Omnibus (GEO) associated with tumor-induced epileptogenesis and applied gene set enrichment analysis (GSEA) on these data sets and performed candidate transcription factor (TF) selection. As a result, 68 upregulated pathways, including the extracellular matrix (ECM)-receptor interaction $(\mathrm{P}=0.004)$ and peroxisome proliferator-activated receptor (PPAR) signaling pathways $(\mathrm{P}=0.045)$, as well as 4 downregulated pathways, including the GnRH signaling pathway $(\mathrm{P}=0.029)$ and gap junction $(\mathrm{P}=0.034)$ were identified as epileptogenesis-related pathways. The majority of these pathways identified have been previously reported and our results were in accordance with those reports. However, some of these pathways identified were novel. Finally, co-expression networks of the related pathways were constructed with the significant core genes and TFs, such as PPAR- $\gamma$ and phosphatidylethanolamine-binding protein. The results of our study may contribute to the improved understanding of the molecular mechanisms of epileptogenesis on a genome-wide level.
\end{abstract}

Correspondence to: Dr Kan He, Center for Stem Cell and Translational Medicine, School of Life Sciences, Anhui University, 111 Jiulong Road, Hefei, Anhui 230601, P.R. China

E-mail: hekan803@gmail.com

${ }^{*}$ Contributed equally

Key words: epilepsy, pathway, gene set enrichment analysis, peroxisome proliferator-activated receptor

\section{Introduction}

Epilepsy is a common chronic neurological disorder characterized by epileptic seizures, which vary in their duration. These episodes can range from brief and nearly undetectable seizures to long periods of vigorous shaking $(1,2)$. In epilepsy, seizures tend to recur, and have no immediate underlying cause, while seizures that occur due to a specific cause are not deemed to represent epilepsy (3). In the majority of cases, the cause of epilepsy is unknown, although some individuals develop epilepsy as a result of brain injury, stroke, brain cancer or drug and alcohol misuse, among others. Epileptic seizures are the result of excessive and abnormal cortical nerve cell activity in the brain (3).

Genetics are believed to be involved in the majority of cases of epilepsy, either directly or indirectly. Although some of the genes involved affect ion channels, other molecules such as enzymes, gamma-aminobutyric acid (GABA) and G proteincoupled receptors have been identified as single genes in which defects cause epilepsy; however, epilepsy may occur due to the interaction of multiple genes and environmental factors (4). The etiology of epilepsy is superficially thought to be multifactorial; however, the genetic factors that may be involved in the pathogenesis of seizures have not yet been elucidated. To study the gene regulatory networks involved in epilepsy, a variety of genome-wide studies have been performed by different groups using various systems and array platforms. The accumulated functional genomic data are freely available in the database of Gene Expression Omnibus (GEO, http://www.ncbi.nlm.nih.gov/ geo/) $(5,6)$, which provides a golden opportunity for compiling a comprehensive list of genetic factors underlying the etiology of epilepsy. According to the approach of differentially expressed gene analysis (DEGA) for studying gene expression profiles, hundreds of significant genes have been identified to be associated with epilepsy. However, few studies have focused on the associated pathways and transcription factors (TFs), as well as on the co-expression patterns at the multiple pathways level.

In the present study, we employed a microarray dataset of genome-wide gene expression profiling from GEO, which is associated with tumor-induced epileptogenesis. The most well-known method of gene set enrichment analysis (GSEA) was used to analyze the genomic data in order to uncover the 
regulatory mechanisms of human epilepsy caused by brain tumors at the multiple pathways level. GSEA is widely used to analyze gene expression profiles, particularly to identify pre-defined gene sets which exhibit significant differences in expression between samples from the control and treatment groups (7-9). The goal of GSEA is to determine other interesting categories (pathways) where the constituent genes show coordinated changes in expression over the experimental conditions, other than in the form of sets of differentially expressed genes (DEGs). One of the advantages of GSEA is the ability to highlight genes weakly connected to the phenotype through pathway analysis, which may be difficult to detect by using classical univariate statistics (7).

\section{Materials and methods}

Microarray data collection and pre-processing. We searched the GEO database (www.ncbi.nlm.nih.gov/geo/) for gene expression profiling studies related to epilepsy. Data were included in our re-analysis if they met the following criteria: i) the data were genome-wide; ii) the comparison was conducted between samples with epilepsy and controls; iii) complete microarray raw or normalized data were available. Finally, we selected the dataset of GSE32534 for our re-analysis, which was contributed by Niesen et al (10). In this dataset, genome-wide gene expression profiling was conducted using the Affymetrix Human Genome U133 Plus 2.0 Array and the RNA was derived from formalin-fixed paraffin-embedded (FFPE) peritumoral cortex tissue slides from 5-paired (seizure vs. non-seizure) low grade brain tumor patients. There were 5 biological replicates for epilepsy [samples from GSM805925 to GSM805929, marked with epilepsy (EP)-1, EP-2, EP-3, EP-4 and EP-5, respectively] and 5 for the controls [samples from GSM805930 to GSM805934, marked with control (CT)-1, CT-2, CT-3, CT-4 and CT-5, respectively].

For the assessment of the influence of pre-processing on the comparison, data pre-processing was performed using software packages developed in version 2.6.0 of Bioconductor and $\mathrm{R}$ version 2.10.1. Each Affymetrix dataset was background adjusted, normalized and $\log 2$ probe-set intensities were calculated using the Robust Multichip Averaging (RMA) algorithm in Affy package, as previously described (11).

GSEA. Our GSEA of pathways and genes was performed using the Category package in version 2.6.0 of Bioconductor, as previously described (12). The goal of GSEA is to determine whether the members of a gene set ' $S$ ' are randomly distributed throughout the entire reference gene list ' $\mathrm{L}$ ' or are primarily found at the top or bottom of the list. One of the advantages of GSEA is the relative robustness to noise and outliers in the data. In our analysis, the gene sets represented by $<10$ genes were excluded. The t-statistic mean of the genes was computed in each Kyoto Encyclopedia of Genes and Genomes (KEGG) pathway. Using a permutation test 1,000 times, the cut-off of the significance level P-values was selected as 0.05 for the significant pathways related to epilepsy. Accordingly, the significant pathways and genes were then identified under the comparison between the samples with epilepsy and no epilepsy. The following classification of identified pathways was based on the KEGG pathway maps br08901 of BRITE
Functional Hierarchies in the KEGG database (http://www. genome.jp/kegg-bin/get_htext?br08901.keg). The annotation of significant genes in each pathway was performed by using biomaRt package, BioMart v. $0.8 \mathrm{rc} 3$ (version of 0.8 release candidate 3; http://www.biomart.org/). Subsequently, clustering of the groups and genes was performed based on the identified gene expression in each significant pathway using the method of hierarchical clustering with Pearson correlation coefficient.

Regulatory elements (REs) and TFs of co-regulated genes. We further employed a web server termed DiRE (Distant RE of co-expressed genes, http://dire.dcode.org/), based on the enhancer identification (EI) method, to predict common REs for our input genes which have co-function in each identified significantly related pathway (13). It predicts function-specific REs consisting of clusters of specifically-associated transcription factor binding sites (TFBSs), and it also scores the association of individual TFs with the biological function shared by the group of input genes. We selected a random set of 5,000 genes in the human genome (hg18) as the source of background genes. As a result, there are 2 major parameters of our predicted TFs, including TF occurrence which denotes the percentage of candidate RE containing a conserved binding site for a particular TF; and TF importance which denotes the product of TF occurrence and TF weight. As our candidate associated TFs with input gene sets, we selected the cut-off value of TF importance as $>0.05$.

\section{Results and Discussion}

Identification of significant pathways associated with epilepsy. In the study of Niesen et al (10), a number of DEGs between the 2 groups (epilepsy and the control) were identified using both the parametric unpaired Student's t-test (345 probe sets representing 296 genes with fold-changes $\geq 2$ plus $\mathrm{P} \leq 0.05$ ) and the non-parametric rank product $[377$ probe sets representing 344 genes with a false discovery rate (FDR) of $\leq 0.3]$. Seven DEGs, i.e., ClQB, CALCRL, CCR1, KAL1, SLC1A2, SSTR1 and TYRO3 were validated by qRT-PCR. Moreover, the pathway analysis using DAVID bioinformatics resources revealed that these DEGs were mainly enriched in focal adhesion, extracellular matrix (ECM)-receptor interaction and cell adhesion molecule (CAM) pathways.

Compared to the approach of DEGs, the strategy of GSEA we used in this study is likely to be more powerful than conventional single-gene methods in the study of complex diseases in which many genes make subtle contributions. According to our GSEA on the dataset of 10 samples by the comparison of epilepsy to the controls, in total, there were 72 significant pathways associated with epilepsy, whose $P$-values were $<0.05$, including 4 downregulated and 68 upregulated pathways. The 3 main pathways identified by Niesen et al (10) were also included in our results. Moreover, based on the KEGG pathway maps in the database of KEGG (http://www.genome. $\mathrm{jp} / \mathrm{kegg} /$ ), these 72 significant pathways could be mainly mapped into 6 functional classes, including cellular processes, environmental information processing, genetic information processing, human diseases, as well as metabolism and organismal systems. The details of the involved pathways in each class are presented in Tables I-V. The details of the associated 


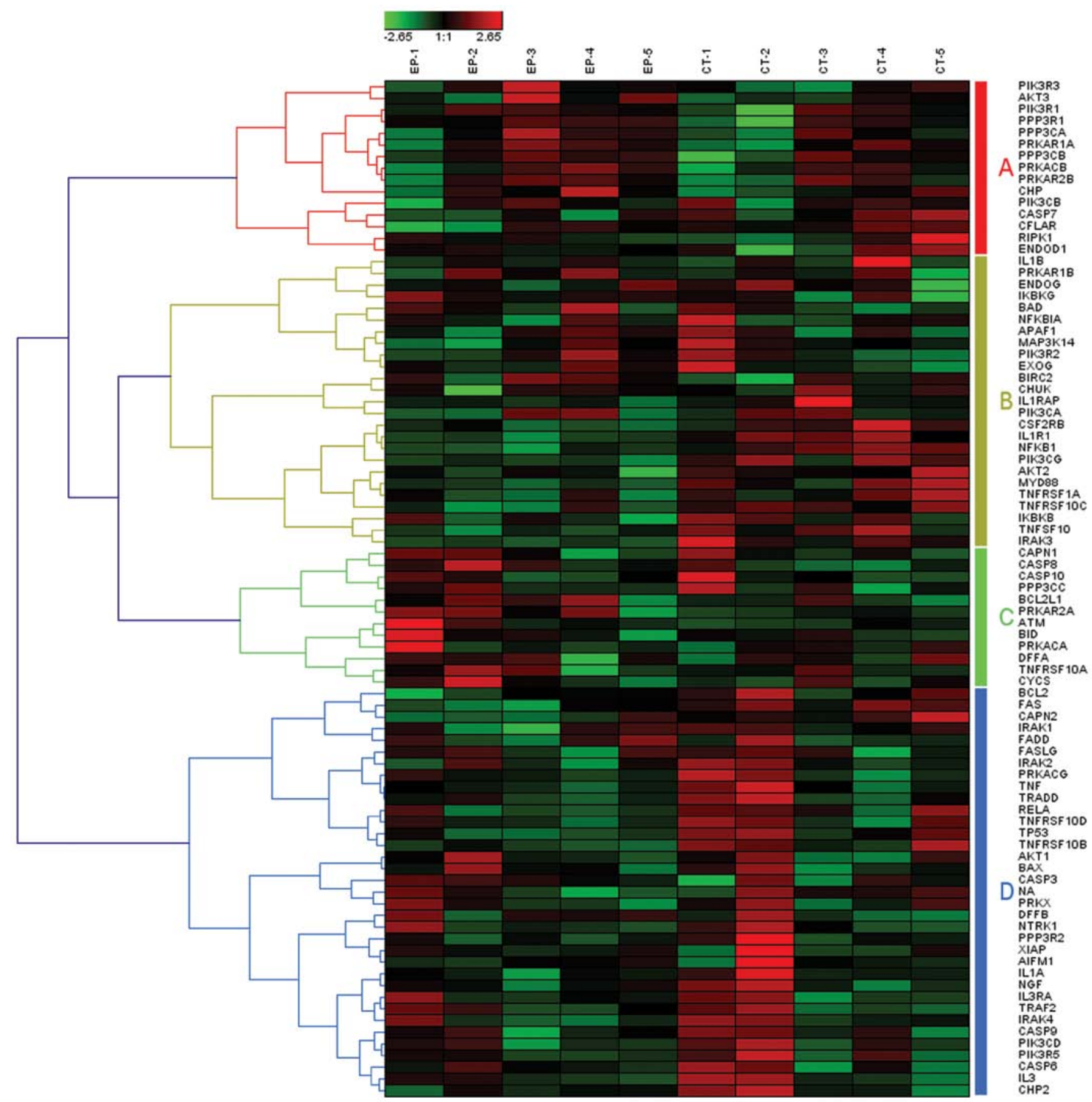

Figure 1. Hierarchical clustering of genes involved in the pathway of apoptosis related to epilepsy. In total, there were 87 genes involved in the pathway of apoptosis related to epilepsy, which may be clustered into 4 gene set groups (groups A-D) based on hierarchical clustering with Pearson correlation coefficient. Heatmap of 87 genes involved in the pathway of apoptosis in 5 epilepsy samples (from EP-1 to EP-5) and 5 control samples (non-epilepsy, from CT-1 to CT-5) is shown. EP, epilepsy; CT, control.

genes in each significant pathway are available upon request (data not shown), including the information on probe set id and gene symbol.

In the functional class of cellular processes, there was 1 significantly downregulated and 8 significantly upregulated pathways associated with epilepsy (Table I). These pathways were involved in cell communication, cell growth and death, cell motility, as well as transport and catabolism. Among these, apoptosis was one of the most significant pathways $(\mathrm{P}<0.001)$, which was classified into the functional group of cell growth and death. As is known, high-frequency stimulation (HFS) of the hippocampus may be a promising method in the treatment of epilepsy. It has been indicated that hippocampal HFS can protect hippocampal neurons against kainic acid (KA) neurotoxicity, and that the neuroprotective effects of HFS may be mediated through the inhibition of apoptosis (14). In total, there were 87 involved genes in the pathway of apoptosis related to epilepsy, which may be clustered into 4 gene set groups based on hierarchical clustering with Pearson correlation coefficient (Fig. 1, groups A-D).

There were 4 and 3 significantly upregulated pathways in the functional class of environmental and genetic infor- 
Table I. Significant pathways associated with epilepsy in the functional class of cellular processes.

\begin{tabular}{|c|c|c|c|c|}
\hline Pathways & Map B & P-value & No. of genes & No. of TFs \\
\hline 04510: Focal adhesion & Cell communication & 0.009 & 199 & 33 \\
\hline $04540^{\text {a. }}$ Gap junction & Cell communication & 0.034 & 86 & 27 \\
\hline 04210: Apoptosis & Cell growth and death & $<0.001$ & 87 & 28 \\
\hline 04115: p53 signaling pathway & Cell growth and death & 0.018 & 68 & 31 \\
\hline 04110: Cell cycle & Cell growth and death & 0.035 & 122 & 37 \\
\hline 04810: Regulation of actin cytoskeleton & Cell motility & 0.048 & 211 & 37 \\
\hline 04146: Peroxisome & Transport and catabolism & $<0.001$ & 78 & 29 \\
\hline 04142: Lysosome & Transport and catabolism & 0.014 & 111 & 26 \\
\hline 04145: Phagosome & Transport and catabolism & 0.031 & 141 & 29 \\
\hline
\end{tabular}

${ }^{\text {a}}$ Represents the significantly downregulated pathways associated with epilepsy. TFs, transcription factors.

Table II. Significant pathways associated with epilepsy in the functional class of environmental and genetic information processing.

\begin{tabular}{|c|c|c|c|c|}
\hline Pathways & Map B & P-value & No. of genes & No. of TFs \\
\hline 04512: ECM-receptor interaction & Signaling molecules and interaction & 0.004 & 84 & 28 \\
\hline 04514: Cell adhesion molecules (CAMs) & Signaling molecules and interaction & 0.020 & 128 & 41 \\
\hline 02010: ABC transporters & Membrane transport & 0.030 & 43 & 17 \\
\hline 04630: Jak-STAT signaling pathway & Signal transduction & 0.046 & 153 & 33 \\
\hline 03020: RNA polymerase & Transcription & 0.008 & 27 & 20 \\
\hline 03430: Mismatch repair & Replication and repair & 0.031 & 21 & 20 \\
\hline 03030: DNA replication & Replication and repair & 0.048 & 35 & 11 \\
\hline
\end{tabular}

TFs, transcription factors; ECM, extracellular matrix.

mation processing, respectively which were associated with epilepsy (Table II). The environmental information processing pathways of ECM-receptor interaction $(\mathrm{P}=0.004)$ and CAMs $(\mathrm{P}=0.020)$ were related to the functions of signaling molecules and interaction, and the $\mathrm{ABC}$ transporter pathway $(\mathrm{P}=0.030)$ was related to the function of membrane transport, and the Jak-STAT signaling pathway $(\mathrm{P}=0.046)$ was related to the function of signal transduction. The genetic information processing pathways of mismatch repair $(\mathrm{P}=0.031)$ and DNA replication $(\mathrm{P}=0.048)$ were related to the functions of replication and repair, and the RNA polymerase pathway $(\mathrm{P}=0.008)$ was transcription related. Among these, the pathway of ECM-receptor interaction was the most significant in this class, which has also been identified as one of the DEG enriched pathways by Niesen et al (10). The ECM is known to regulate important processes in neuronal cell development, activity and growth. The remodeling of the ECM during both development and following injury to the central nervous system has been shown to affect neuronal guidance, synaptic plasticity and their regenerative responses. The functions and potential therapeutic value of several key ECM molecules in epileptogenesis and dementia have been extensively investigated in previous studies $(15,16)$. The 84 genes involved in the pathway of ECM-receptor interaction may also be clustered into 4 gene set groups (Fig. 2, groups A-D).
Twenty-five significantly associated pathways were classified into the functional class of human diseases, including 5 upregulated and 1 downregulated cancer-related pathways, 4 upregulated cardiovascular disease-related pathways, 1 upregulated endocrine and metabolic disease-related pathway, 6 upregulated immune disease-related pathways and 8 upregulated infectious disease-related pathways (Table III). It has been reported that cardiac changes may accompany epilepsy, which may lead to significant seizure-associated cardiac performance decreases (17). These data are consistent with the results from our study which identidied 4 significant cardiovascular disease-related pathways associated with epilepsy. Among these, the pathway of hypertrophic cardiomyopathy (HCM) was one of the most significant cardiovascular disease-related pathways $(\mathrm{P}=0.004)$. The association between these 2 diseases has been revealed in a clinical case report (18). In total, there were 83 genes involved in the pathway of HCM associated with epilepsy, which may be clustered into 4 gene set groups (Fig. 3, groups A-D).

In the functional class of metabolism, there were 14 significantly upregulated pathways associated with epilepsy (Table IV). These were involved in 6 different types of metabolism, including amino acid metabolism, carbohydrate metabolism, glycan biosynthesis and metabolism, lipid metabolism, metabolism of co-factors and vitamins, as well as 


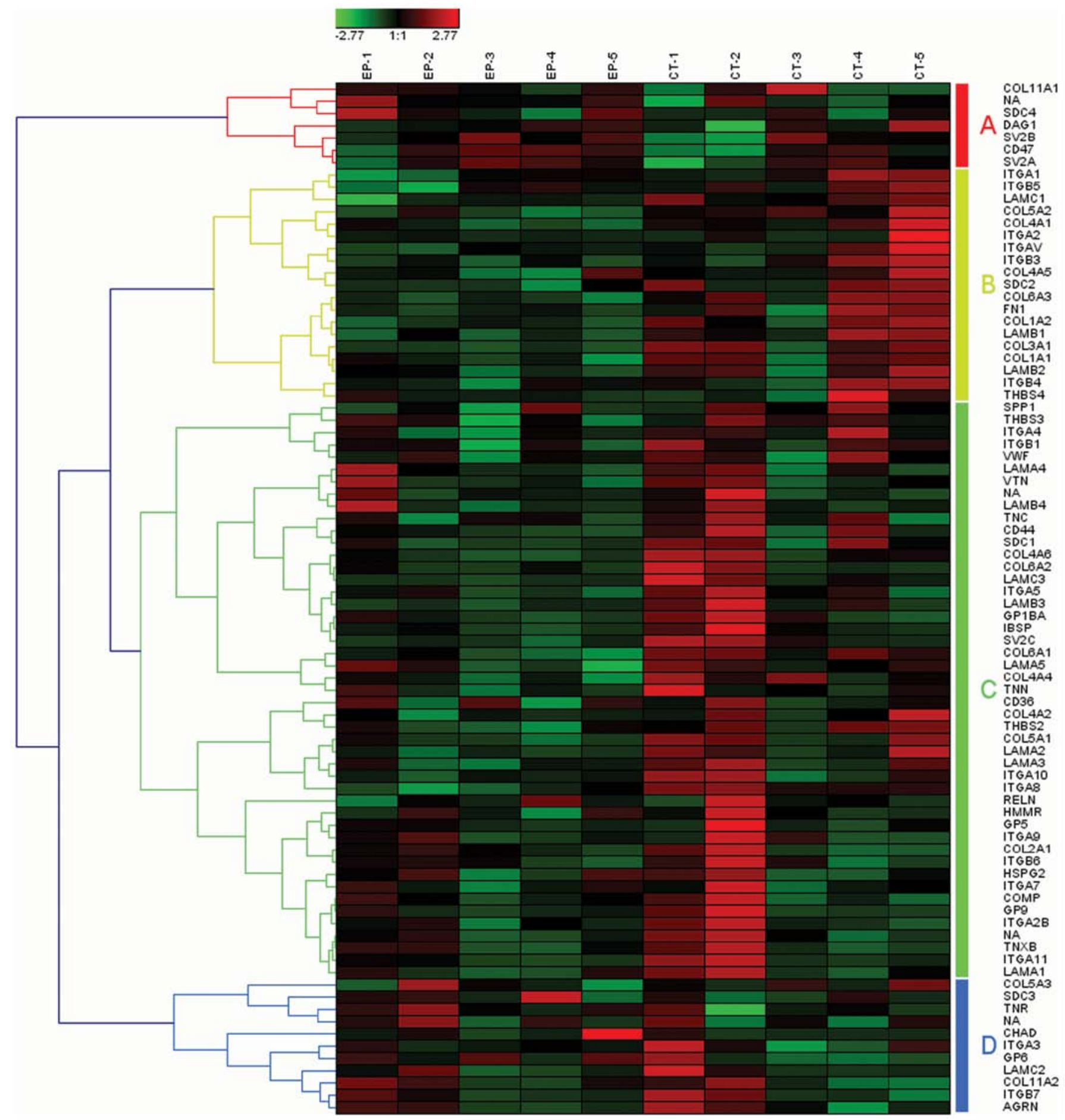

Figure 2. Hierarchical clustering of genes involved in the pathway of extracellular matrix (ECM)-receptor interaction related to epilepsy. In total, there were 84 genes involved in the pathway of ECM-receptor interaction related to epilepsy, which may be clustered into 4 gene set groups (groups A-D) based on hierarchical clustering with Pearson correlation coefficient. Heatmap of 84 genes involved in the pathway of ECM-receptor interaction in 5 epilepsy samples (from EP-1 to EP-5) and 5 control samples (non-epilepsy, from CT-1 to CT-5) is shown. EP, epilepsy; CT, control.

nucleotide metabolism. Among these, the pentose phosphate pathway was one of the most significant pathways $(\mathrm{P}=0.007)$, which was classified into the functional group of carbohydrate metabolism. A variety of observations suggested that decreasing glycolysis and increasing the levels of reduced glutathione, generated by the metabolism of glucose through the pentose phosphate pathway, may have an anticonvulsant effect. Fructose-1,6-bisphosphate (F1,6BP) may have anticon- vulsant activity in models of acute seizures in adult rats, which shifts the metabolism of glucose from glycolysis to the pentose phosphate pathway (19). The total of 25 genes involved in the pentose phosphate pathway may be clustered into 3 gene set groups (Fig. 4, groups A-C).

In the last functional class of organismal systems, there were 2 significantly downregulated and 15 significantly upregulated pathways associated with epilepsy (Table V). These 


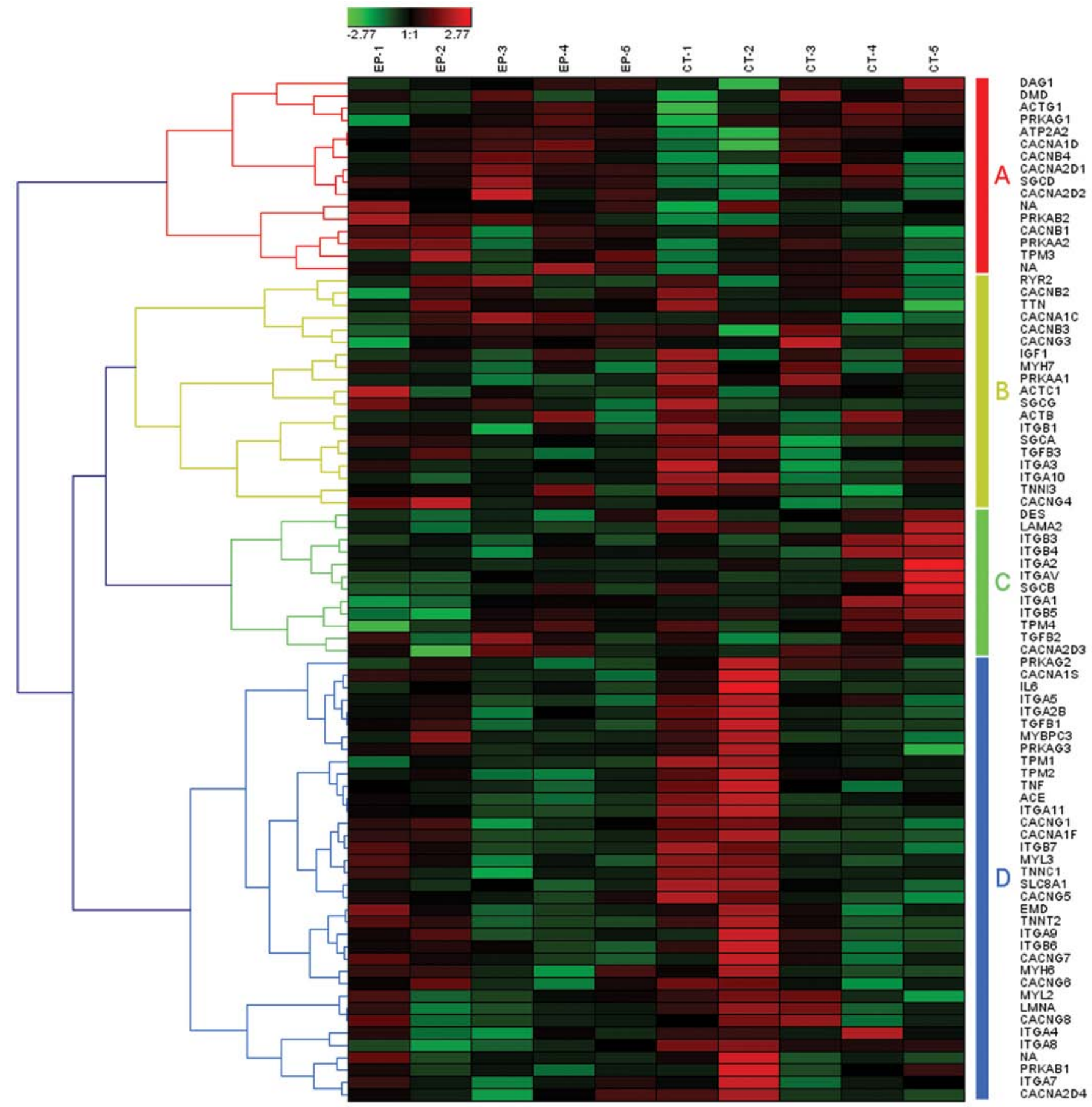

Figure 3. Hierarchical clustering of genes involved in the pathway of hypertrophic cardiomyopathy (HCM) related to epilepsy. In total, there were 83 genes involved in the pathway of HCM related to epilepsy, which may be clustered into 4 gene set groups (groups A-D) based on hierarchical clustering with Pearson correlation coefficient. Heatmap of 83 genes involved in the pathway of HCM in 5 epilepsy samples (from EP-1 to EP-5) and 5 control samples (non-epilepsy, from CT-1 to CT-5) is shown. EP, epilepsy; CT, control.

were involved in development, the nervous system, the endocrine system, as well as the immune system. Among these, the peroxisome proliferator-activated receptor (PPAR) signaling pathway was one of the most associated pathways $(\mathrm{P}=0.045)$, which was classified into the functional group of endocrine system. PPAR- $\gamma /$ mitochondrial uncoupling protein 2 signaling has been reported to protect neurons against seizure-induced neuronal cell death in the hippocampus following experimental status epilepticus (20). The activation of the mammalian target of rapamycin or PPAR- $\gamma$ pathways has been considered as one of the pre-clinical models for the anti-epileptogenic activity of a diverse range of potential therapies (21). Furthermore, there were 68 genes in total which were involved in the PPAR signaling pathway associated with epilepsy, which may be clustered into 4 gene set groups (Fig. 5, groups A-D).

Candidate TF selection related to epilepsy. To predict the TFs potentially involved in the regulation of epilepsy, we 
Table III. Significant pathways associated with epilepsy in the functional class of human diseases.

\begin{tabular}{|c|c|c|c|c|}
\hline Pathways & Map B & P-value & No. of genes & No. of TFs \\
\hline 05222: Small cell lung cancer & Cancers & 0.008 & 85 & 38 \\
\hline $05213^{\mathrm{a}}$ : Endometrial cancer & Cancers & 0.008 & 52 & 30 \\
\hline 05212: Pancreatic cancer & Cancers & 0.011 & 69 & 40 \\
\hline 05218: Melanoma & Cancers & 0.012 & 71 & 40 \\
\hline 05200: Pathways in cancer & Cancers & 0.022 & 323 & 30 \\
\hline 05219: Bladder cancer & Cancers & 0.023 & 41 & 16 \\
\hline 05410: Hypertrophic cardiomyopathy (HCM) & Cardiovascular diseases & 0.004 & 83 & 37 \\
\hline $\begin{array}{l}\text { 05412: Arrhythmogenic right ventricular } \\
\text { cardiomyopathy (ARVC) }\end{array}$ & Cardiovascular diseases & 0.004 & 74 & 33 \\
\hline 05416: Viral myocarditis & Cardiovascular diseases & 0.034 & 64 & 21 \\
\hline 05414: Dilated cardiomyopathy & Cardiovascular diseases & 0.049 & 90 & 37 \\
\hline 04940: Type I diabetes mellitus & Endocrine and metabolic diseases & 0.013 & 39 & 24 \\
\hline 05323: Rheumatoid arthritis & Immune diseases & 0.004 & 83 & 19 \\
\hline 05322: Systemic lupus erythematosus & Immune diseases & 0.013 & 82 & 24 \\
\hline 05320: Autoimmune thyroid disease & Immune diseases & 0.013 & 47 & 20 \\
\hline 05330: Allograft rejection & Immune diseases & 0.013 & 33 & 14 \\
\hline 05332: Graft-versus-host disease & Immune diseases & 0.013 & 33 & 21 \\
\hline 05310: Asthma & Immune diseases & 0.020 & 26 & 27 \\
\hline 05100: Bacterial invasion of epithelial cells & Infectious diseases: Bacterial & 0.009 & 70 & 36 \\
\hline 05150: Staphylococcus aureus infection & Infectious diseases: Bacterial & 0.013 & 48 & 19 \\
\hline 05146: Amoebiasis & Infectious diseases: Parasitic & $<0.001$ & 106 & 25 \\
\hline 05145: Toxoplasmosis & Infectious diseases: Parasitic & 0.008 & 120 & 35 \\
\hline 05140: Leishmaniasis & Infectious diseases: Parasitic & 0.008 & 66 & 25 \\
\hline 05144: Malaria & Infectious diseases: Parasitic & 0.024 & 48 & 28 \\
\hline $\begin{array}{l}\text { 05142: Chagas disease } \\
\text { (American trypanosomiasis) }\end{array}$ & Infectious diseases: Parasitic & 0.036 & 101 & 41 \\
\hline 05160: Hepatitis C & Infectious diseases: Viral & 0.018 & 131 & 38 \\
\hline
\end{tabular}

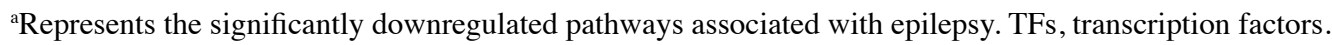

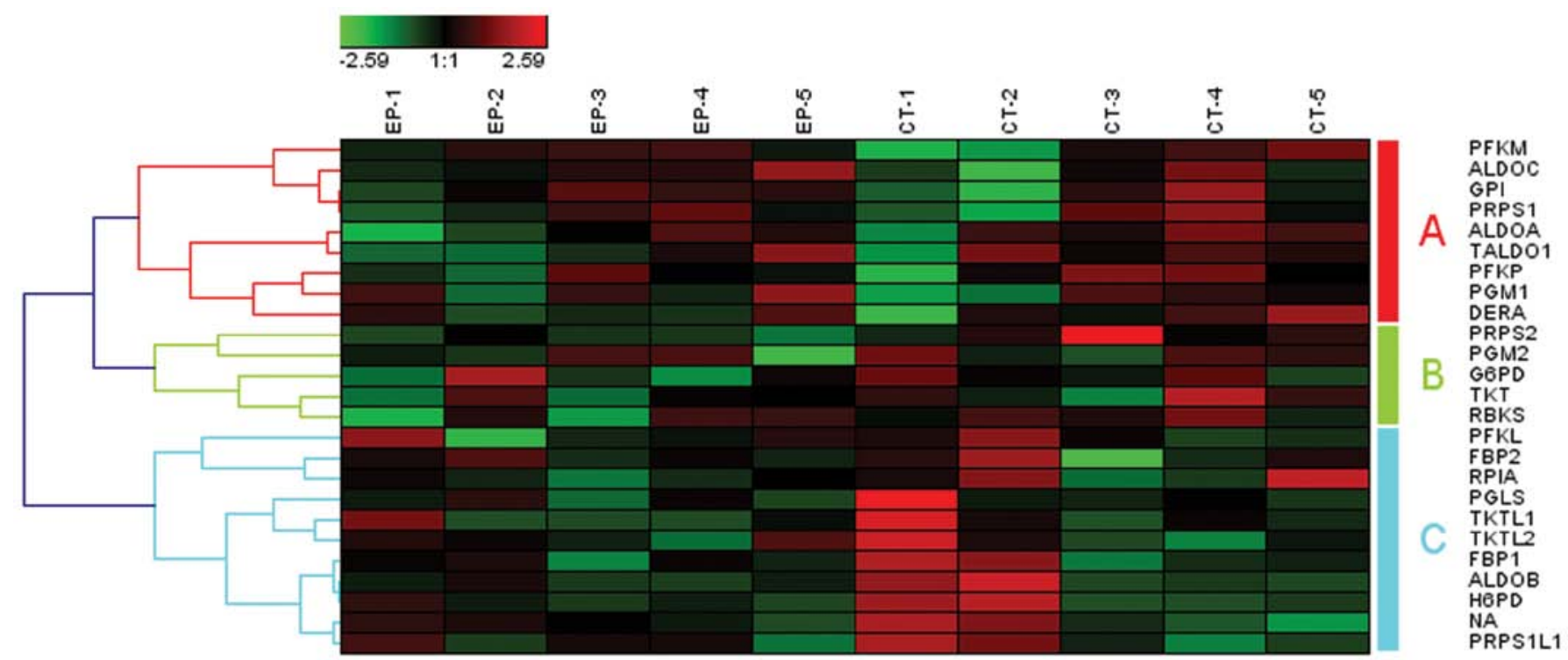

Figure 4. Hierarchical clustering of genes involved in pentose phosphate pathway related to epilepsy. In total, there were 25 genes involved in the pentose phosphate pathway related to epilepsy, which may be clustered into 3 gene set groups (groups A-C) based on hierarchical clustering with Pearson correlation coefficient from group of A to C. Heatmap of 25 genes involved in the pentose phosphate pathway in 5 epilepsy samples (from EP-1 to EP-5) and 5 control samples (non-epilepsy, from CT-1 to CT-5) is shown. EP, epilepsy; CT, control. 
Table IV. Significant pathways associated with epilepsy in the functional class of metabolism.

\begin{tabular}{|c|c|c|c|c|}
\hline Pathways & Map B & P-value & No. of genes & No. of TFs \\
\hline 00360: Phenylalanine metabolism & Amino acid metabolism & 0.031 & 17 & 26 \\
\hline 00380: Tryptophan metabolism & Amino acid metabolism & 0.039 & 42 & 21 \\
\hline 00030: Pentose phosphate pathway & Carbohydrate metabolism & 0.007 & 25 & 39 \\
\hline $\begin{array}{l}\text { 00520: Amino sugar and nucleotide } \\
\text { sugar metabolism }\end{array}$ & Carbohydrate metabolism & 0.018 & 46 & 29 \\
\hline 00010: Glycolysis/gluconeogenesis & Carbohydrate metabolism & 0.024 & 63 & 25 \\
\hline 00511: Other glycan degradation & Glycan biosynthesis and metabolism & 0.014 & 16 & 22 \\
\hline $\begin{array}{l}\text { 00604: Glycosphingolipid biosynthesis - } \\
\text { ganglio series }\end{array}$ & Glycan biosynthesis and metabolism & 0.043 & 15 & 26 \\
\hline 00071: Fatty acid metabolism & Lipid metabolism & 0.021 & 42 & 22 \\
\hline 00100: Steroid biosynthesis & Lipid metabolism & 0.032 & 18 & 21 \\
\hline $\begin{array}{l}\text { 00860: Porphyrin and chlorophyll } \\
\text { metabolism }\end{array}$ & Metabolism of cofactors and vitamins & 0.009 & 30 & 23 \\
\hline $\begin{array}{l}\text { 00770: Pantothenate and CoA } \\
\text { biosynthesis }\end{array}$ & Metabolism of cofactors and vitamins & 0.013 & 16 & 20 \\
\hline 00480: Glutathione metabolism & Metabolism of other amino acids & 0.022 & 47 & 19 \\
\hline 00230: Purine metabolism & Nucleotide metabolism & $<0.001$ & 155 & 26 \\
\hline 00240: Pyrimidine metabolism & Nucleotide metabolism & 0.029 & 92 & 43 \\
\hline
\end{tabular}

TFs, transcription factors.

Table V. Significant pathways associated with epilepsy in the functional class of organismal systems.

\begin{tabular}{|c|c|c|c|c|}
\hline Pathways & Map B & P-value & No. of genes & No. of TFs \\
\hline 04380: Osteoclast differentiation & Development & 0.004 & 125 & 45 \\
\hline $04730^{\text {a: }}$ : Long-term depression & Nervous system & 0.004 & 65 & 46 \\
\hline 04920: Adipocytokine signaling pathway & Endocrine system & 0.027 & 67 & 31 \\
\hline 04912ª GnRH signaling pathway & Endocrine system & 0.029 & 94 & 25 \\
\hline 03320: PPAR signaling pathway & Endocrine system & 0.045 & 68 & 21 \\
\hline 04650: Natural killer cell mediated cytotoxicity & Immune system & 0.004 & 125 & 31 \\
\hline 04670: Leukocyte transendothelial migration & Immune system & 0.009 & 113 & 46 \\
\hline 04610: Complement and coagulation cascades & Immune system & 0.013 & 67 & 25 \\
\hline 04672: Intestinal immune network for IgA production & Immune system & 0.013 & 44 & 30 \\
\hline 04640: Hematopoietic cell lineage & Immune system & 0.014 & 84 & 31 \\
\hline 04612: Antigen processing and presentation & Immune system & 0.017 & 63 & 21 \\
\hline 04666: Fc gamma R-mediated phagocytosis & Immune system & 0.018 & 90 & 31 \\
\hline 04620: Toll-like receptor signaling pathway & Immune system & 0.024 & 99 & 30 \\
\hline 04662: B cell receptor signaling pathway & Immune system & 0.028 & 74 & 43 \\
\hline 04621: NOD-like receptor signaling pathway & Immune system & 0.032 & 58 & 20 \\
\hline 04623: Cytosolic DNA-sensing pathway & Immune system & 0.033 & 53 & 17 \\
\hline 04660: T cell receptor signaling pathway & Immune system & 0.048 & 108 & 35 \\
\hline
\end{tabular}

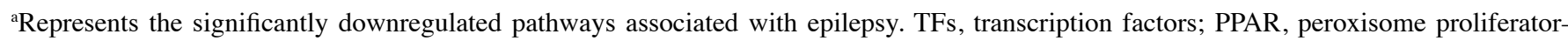
activated receptor.

performed the analysis of TFBSs and the prediction of TFs using the significant genes in each identified pathway. Based $n$ the cut-off value of TF importance, we identified the candidate TFs related to epilepsy with potential target genes which were co-regulated in each of the 72 pathways identified. The details are available upon request.

Among these, the TF of PPAR- $\gamma$ appeared in several pathways, including fatty acid metabolism, pyrimidine 


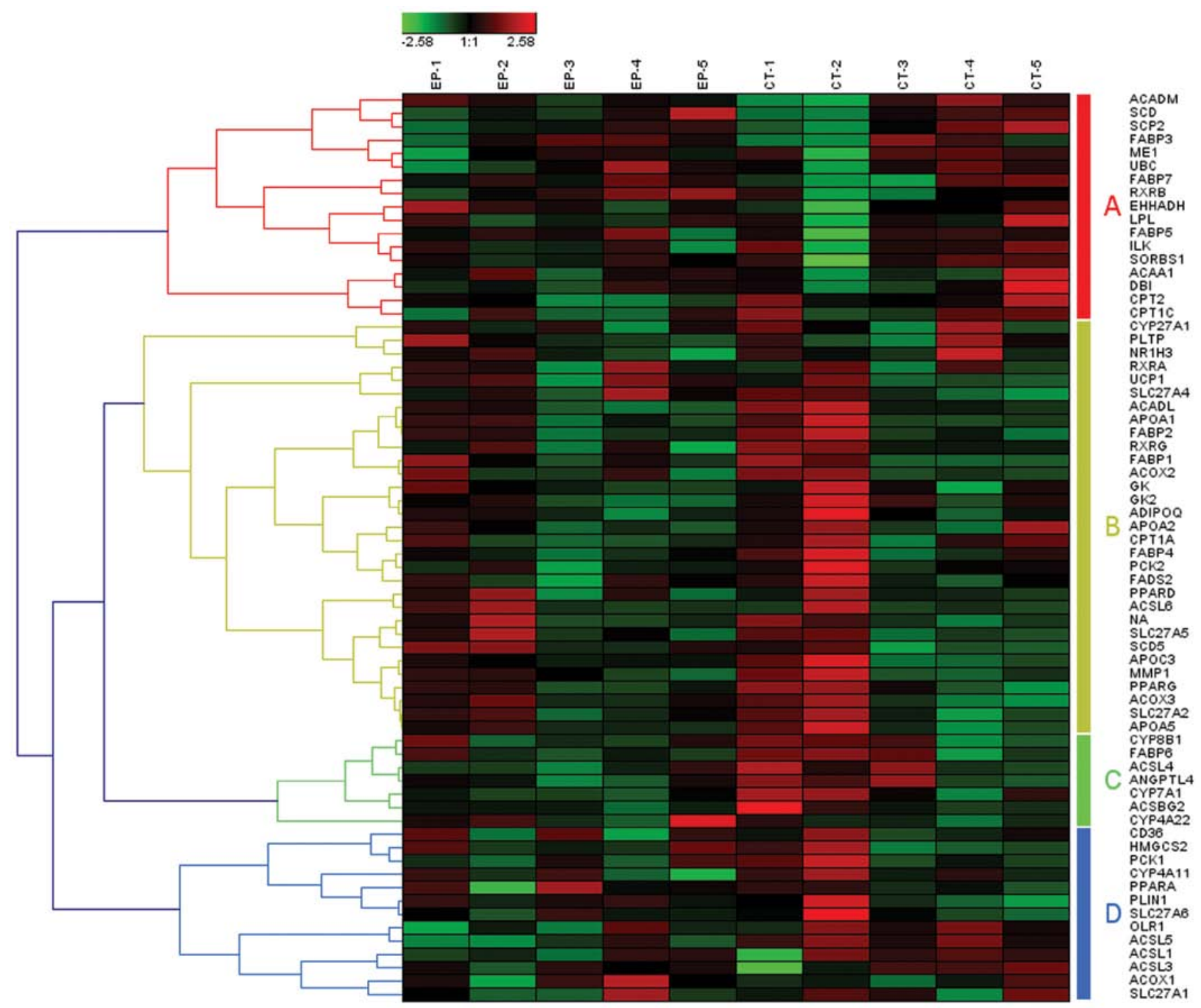

Figure 5. Hierarchical clustering of genes involved in peroxisome proliferato-activated receptor (PPAR) signaling pathway related to epilepsy. In total, there were 68 genes involved in the PPAR signaling pathway related to epilepsy, which may be clustered into 4 gene set groups (groups A-C) based on hierarchical clustering with Pearson correlation coefficient. Heatmap of 68 genes involved in the pentose phosphate pathway in 5 epilepsy samples (from EP-1 to EP-5) and 5 control samples (non-epilepsy, from CT-1 to CT-5) is shown. EP, epilepsy; CT, control.

metabolism, the PPAR signaling pathway, lysosome, amoebiasis and melanoma (data available upon request). PPARs belong to the nuclear hormone receptor superfamily. They play critical physiological roles as lipid sensors and regulators of lipid metabolism and are activated by fatty acids (22). Previous studies have shown that PPAR- $\gamma$ has anti-inflammatory effects in seizure animal models $(20,23)$. The anticonvulsant effects of acute pioglitazone on pentylenetetrazol (PTZ)-induced seizures in mice have been demonstrated to be mediated through the PPAR- $\gamma$ receptor-mediated pathway (24). One of the PPAR- $\gamma$ agonists, rosiglitazone, has been proven to protect the central nervous system from oxidative damage in epileptic rats, which may be a potential neuroprotective agent for epilepsy (25). Our data also support the idea that PPAR- $\gamma$ may be one of the essential targets for the management of epilepsy due to brain tumors.
Another essential TF of the phosphatidylethanolaminebinding protein (PEBP) was identified in 3 of 4 downregulated pathways, including the GnRH signaling pathway, gap junction and long-term depression pathways. PEBP is alternatively named Raf-1 kinase inhibitor protein, the precursor of the hippocampal cholinergic neurostimulating peptide (HCNP) corresponding to its natural N-terminal fragment, which has been previously described to be released by hippocampal neurons (26-28). The crystal structure of human PEBP (hPEBP) suggests that the ligand-binding site may accommodate the phosphate head groups of membrane lipids, therefore allowing the protein to adhere to the inner leaf of bilipid membranes where it would be ideally positioned to relay signals from the membrane to the cytoplasm (29). With the previous evidence of calpain dysregulation in Alzheimer's disease (AD), PEBP has been confirmed as a novel in vitro 
and in situ calpain substrate using an in vitro proteomics approach or serial analysis of gene expression (SAGE) $(30,31)$. In particular, during brain development, the N-terminal part of mammalian PEBP has been reported to be specifically cleaved and the resulting 11 amino acid peptides may stimulate the growth and activity of acetylcholinergic neurons (32).

\section{Acknowledgements}

We acknowledge financial support by the Scientific Research Foundation and Academic and Technology Leaders Introduction Project, and the 211 project of Anhui University (10117700023).

\section{References}

1. Chang BS and Lowenstein DH: Epilepsy. N Engl J Med 349: 1257-1266, 2003.

2. Magiorkinis E, Sidiropoulou $\mathrm{K}$ and Diamantis A: Hallmarks in the history of epilepsy: epilepsy in antiquity. Epilepsy Behav 17: 103-108, 2010.

3. Fisher RS, van Emde Boas W, Blume W, et al: Epileptic seizures and epilepsy: definitions proposed by the International League Against Epilepsy (ILAE) and the International Bureau for Epilepsy (IBE). Epilepsia 46: 470-472, 2005.

4. Mulley JC, Scheffer IE, Harkin LA, Berkovic SF and Dibbens LM: Susceptibility genes for complex epilepsy. Hum Mol Genet 14: R243-R249, 2005.

5. Edgar R, Domrachev M and Lash AE: Gene Expression Omnibus: NCBI gene expression and hybridization array data repository. Nucleic Acids Res 30: 207-210, 2002.

6. Barrett T, Wilhite SE, Ledoux P, et al: NCBI GEO: archive for functional genomics data sets - update. Nucleic Acids Res 41: D991-D995, 2013.

7. Subramanian A, Tamayo P, Mootha VK, et al: Gene set enrichment analysis: a knowledge-based approach for interpreting genome-wide expression profiles. Proc Natl Acad Sci USA 102: 15545-15550, 2005.

8. He K, Chen Z, Ma Y and Pan Y: Identification of high-copperresponsive target pathways in Atp7b knockout mouse liver by GSEA on microarray data sets. Mamm Genome 22: 703-713, 2011.

9. Zhao H, Wang Q, Bai C, He K and Pan Y: A cross-study gene set enrichment analysis identifies critical pathways in endometriosis. Reprod Biol Endocrinol 7: 94, 2009.

10. Niesen CE, Xu J, Fan X, et al: Transcriptomic profiling of human peritumoral neocortex tissues revealed genes possibly involved in tumor-induced epilepsy. PLoS One 8: e56077, 2013.

11. Gautier L, Cope L, Bolstad BM and Irizarry RA: affy - analysis of Affymetrix GeneChip data at the probe level. Bioinformatics 20: 307-315, 2004

12. Chiaretti S, Li X, Gentleman R, et al: Gene expression profile of adult T-cell acute lymphocytic leukemia identifies distinct subsets of patients with different response to therapy and survival. Blood 103: 2771-2778, 2004.

13. Gotea V and Ovcharenko I: DiRE: identifying distant regulatory elements of co-expressed genes. Nucleic Acids Res 36 : W133-W139, 2008.

14. Chen N, Gao Y, Yan N, et al: High-frequency stimulation of the hippocampus protects against seizure activity and hippocampal neuronal apoptosis induced by kainic acid administration in macaques. Neuroscience 256: 370-378, 2014.

15. Soleman S, Filippov MA, Dityatev A and Fawcett JW: Targeting the neural extracellular matrix in neurological disorders. Neuroscience 253: 194-213, 2013.
16. Heck N, Garwood J, Loeffler JP, Larmet Y and Faissner A: Differential upregulation of extracellular matrix molecules associated with the appearance of granule cell dispersion and mossy fiber sprouting during epileptogenesis in a murine model of temporal lobe epilepsy. Neuroscience 129: 309-324, 2004.

17. Naggar I, Lazar J, Kamran H, Orman R and Stewart M: Relation of autonomic and cardiac abnormalities to ventricular fibrillation in a rat model of epilepsy. Epilepsy Res 108: 44-56, 2014.

18. Iniesta I, Yotti R and Garcia-Pastor A: Transient loss of consciousness with convulsions in two young adults with potentially fatal underlying heart disease: syncope versus seizures. BMJ Case Rep 2009: pii: bcr06.2008.0285, 2009.

19. Lian XY, Khan FA and Stringer JL: Fructose-1,6-bisphosphate has anticonvulsant activity in models of acute seizures in adult rats. J Neurosci 27: 12007-12011, 2007.

20. Chuang YC, Lin TK, Huang HY, et al: Peroxisome proliferatoractivated receptors $\gamma /$ mitochondrial uncoupling protein 2 signaling protects against seizure-induced neuronal cell death in the hippocampus following experimental status epilepticus. J Neuroinflammation 9: 184, 2012

21. Kaminski RM, Rogawski MA and Klitgaard H: The potential of antiseizure drugs and agents that act on novel molecular targets as antiepileptogenic treatments. Neurotherapeutics 11: 385-400, 2014.

22. Hihi AK, Michalik L and Wahli W: PPARs: transcriptional effectors of fatty acids and their derivatives. Cell Mol Life Sci 59: 790-798, 2002.

23. Fuenzalida K, Quintanilla R, Ramos $\mathrm{P}$, et al: Peroxisome proliferator-activated receptor gamma up-regulates the Bcl-2 anti-apoptotic protein in neurons and induces mitochondrial stabilization and protection against oxidative stress and apoptosis. J Biol Chem 282: 37006-37015, 2007.

24. Adabi Mohazab R, Javadi-Paydar M, Delfan B and Dehpour AR: Possible involvement of PPAR-gamma receptor and nitric oxide pathway in the anticonvulsant effect of acute pioglitazone on pentylenetetrazole-induced seizures in mice. Epilepsy Res 101: 28-35, 2012.

25. Yu X, Shao XG, Sun H, et al: Activation of cerebral peroxisome proliferator-activated receptors gamma exerts neuroprotection by inhibiting oxidative stress following pilocarpine-induced status epilepticus. Brain Res 1200: 146-158, 2008.

26. Goumon Y, Angelone T, Schoentgen F, et al: The hippocampal cholinergic neurostimulating peptide, the $\mathrm{N}$-terminal fragment of the secreted phosphatidylethanolamine-binding protein, possesses a new biological activity on cardiac physiology. J Biol Chem 279: 13054-13064, 2004.

27. Yeung K, Seitz T, Li S, et al: Suppression of Raf-1 kinase activity and MAP kinase signalling by RKIP. Nature 401: 173-177, 1999.

28. Frayne J, Ingram C, Love S and Hall L: Localisation of phosphatidylethanolamine-binding protein in the brain and other tissues of the rat. Cell Tissue Res 298: 415-423, 1999.

29. Banfield MJ, Barker JJ, Perry AC and Brady RL: Function from structure? The crystal structure of human phosphatidylethanolamine-binding protein suggests a role in membrane signal transduction. Structure 6: 1245-1254, 1998.

30. Chen Q, Wang S, Thompson SN, Hall ED and Guttmann RP: Identification and characterization of PEBP as a calpain substrate. J Neurochem 99: 1133-1141, 2006.

31. George AJ, Gordon L, Beissbarth T, et al: A serial analysis of gene expression profile of the Alzheimer's disease $\mathrm{Tg} 2576$ mouse model. Neurotox Res 17: 360-379, 2010.

32. Vallée BS, Coadou G, Labbé H, Sy D, Vovelle F and Schoentgen F: Peptides corresponding to the N- and C-terminal parts of PEBP are well-structured in solution: new insights into their possible interaction with partners in vivo. J Pept Res 61: 47-57, 2003. 